\title{
Live Demonstration: Performance Evaluation of Electrical Impedance Tomography Systems Using a Color-Coded Full Reference SNR Method
}

\author{
Yu Wu, Dai Jiang, Nazanin Neshatvar, Farnaz Fahimi Hanzaee, and Andreas Demosthenous \\ Department of Electronic and Electrical Engineering, University College London, Torrington Place, London WC1E 7JE, UK \\ e-mail: \{yu.wu.09, d.jiang, a.demosthenous\}@ucl.ac.uk
}

\begin{abstract}
A color-coded full reference signal-to-noise (FR-SNR) method is proposed as a simple and reproducible performance evaluation method for EIT systems. It will be demonstrated with a 16-electrode high-speed EIT system and a resistive phantom. The performance of the EIT system can be altered by artificially introducing non-ideal operating conditions such as low drive current, low ADC resolution, dc offset in the readout front-end and large electrode impedance. The performance under these conditions will be measured and displayed using the FR-SNR method.
\end{abstract}

\section{INTRODUCTION}

Electrical impedance tomography (EIT) as a low-risk and low-cost imaging method has attracted increasing attention in academia and industry for developing real-time imaging devices for biomedical applications. In addition to wearable devices for applications such as respiratory monitoring [1], recent development includes implantable devices for distinguishing neural activities [2] and cell imaging device for visualizing 3D cell cultures [3]. EIT systems have been reported in the literature with different system architectures, circuit implementations, electrode interfaces and image reconstruction algorithms. The methods used to evaluate the performance of these systems are largely different with respect to the test setup, the material of the subject-under-test (SUT) and the metrics for quantifying the performance evaluation. A simple and reproducible methodology towards a universal figure-of-merit (FoM) will greatly benefit the exploitation of EIT hardware specifications for different applications.

This work proposes an objective, full-reference method for evaluating the performance of EIT systems [4]. It differs from existing EIT evaluation methods where e.g. the signal-to-noise ratio (SNR) is calculated to show data variation over multiple measurements and does not fully reflect the EIT image quality. The proposed method first generates an ideal reference image using simulated data obtained from a resistive phantom model, and later computes the image errors between the image acquired using the system-under-test on the same phantom and the ideal reference image. The calculated error which is computed in SNR is displayed using color mapping for easy visual recognition. The image quality is evaluated with both global and regional SNR.

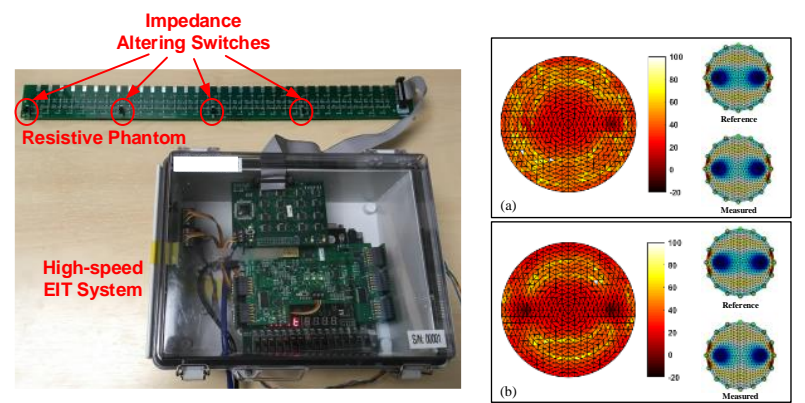

Fig. 1. A 16-electrode EIT system and a resistive phantom in the setup of the proposed demonstration (left), and FR-SNR mapping together with the corresponding EIT images (right).

\section{DEMONSTRATION SETUP AND VISITOR EXPERIENCE}

A 16-electrode high-speed EIT system, a resistive phantom and a laptop for calculating the FR-SNR is provided in the demonstration, as shown in Fig. 1. Visitors can simulate impedance variation in the SUT using four impedance altering switches on the resistive phantom. In addition, to demonstrate the change in FR-SNR with the EIT system in different conditions, visitors can modify the system by: 1) adjusting the injection current; 2) varying the resolution of the digitization in the readout front-end; 3) injecting a small dc offset at the ADC inputs; 4) changing the electrode impedance by adjusting the impedance values on an electrode adaptor board; and 5) modifying the bandwidth of the readout frontend.

\section{REFERENCES}

[1] Y. Wu, D. Jiang, A. Bardill, S. De Gelidi, R. Bayford, and A. Demosthenous, "A high frame rate wearable EIT system using active electrode ASICs for lung respiration and heart rate monitoring," IEEE Trans. Circuits Syst. I Regul. Pap., vol. 65, no. 11, pp. 3810-3820, 2018.

[2] C. A. R. Chapman et al., "Electrode fabrication and interface optimization for imaging of evoked peripheral nervous system activity with electrical impedance tomography (EIT)," J. Neural Eng., vol. 16, no. 1, 2019

[3] Y. Yang, J. Jia, S. Smith, N. Jamil, W. Gamal and P. Bagnaninchi, "A Miniature Electrical Impedance Tomography Sensor and 3-D Image Reconstruction for Cell Imaging," in IEEE Sensors Journal, vol. 17, no. 2, pp. 514-523, 15 Jan.15, 2017.

[4] Y. Wu, D. Jiang, Nazanin Neshatvar, Farnaz Fahimi Hanzaee, and A. Demosthenous, "Towards a universal methodology for performance evaluation of electrical impedance tomography systems using full reference SNR”, IEEE Int. Symp. Circuits and Systems (ISCAS 2020), May 2020. 